The program can be invoked from the command line together with the name of the file or the code (name without extension) of a CIF or CFL file. The program looks first for the existence of a CFL file with the given code, if there is no CFL file it looks for a CIF file.

Alternatively the program can be invoked with the name of a buffer file in which there is a list of CIF or CFL file. Depending on additional arguments the program perform different calculations.

Alternatively, the GUI GBond_Str program can be used directly to convert CIF files to CFL files. Remember that information about the chemical species (ionic oxidation states) is not always included in CIF files, so the user has to include it in the appropriate place in the atom string (see below) if he (she) wants to make bond-valence calculations (alternatively the user can run the program Formal_Charges for getting a proper CFL file).

All it is needed to know about the input files and running the program is explained in the following examples.

\section{Example 1:}

A CIF file, called 99677. cif, exists in the current directory (and there is no file called 99677. Cfl); the program can be invoked as follows:

Current_directory> Bond_Str 99677 <cr>

$<\mathrm{cr}>$ corresponds to carriage return (ENTER key)

The screen output is the following:

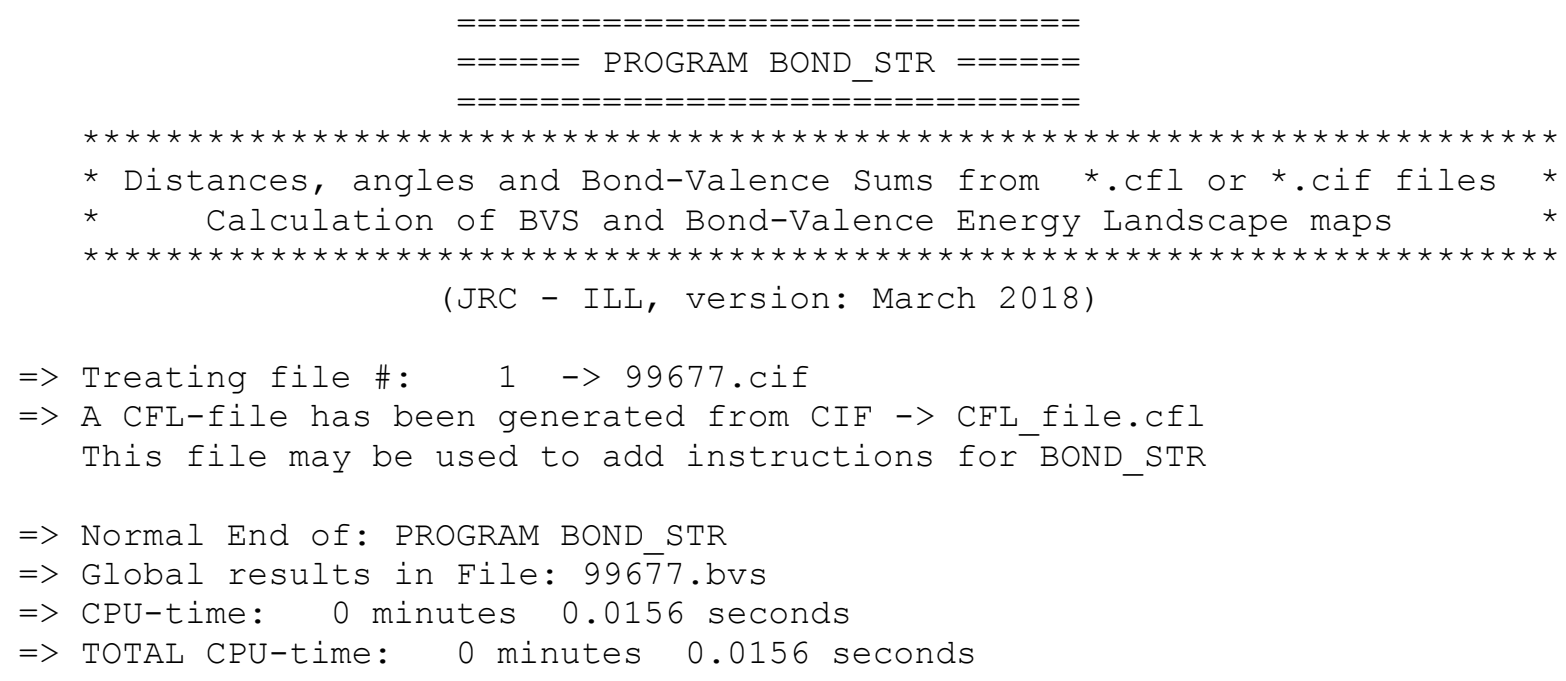

In the input CIF file there is no information to calculate the oxidation state of the different ions, so bond-valence calculations are not performed. Only distances up to 3.2 angstroms are calculated for the current structure. A file called CFL file. $\mathrm{Cfl}$ has also been generated and the user can include the necessary information to perform the complete calculations in further 
runs (using the CFL file instead of the CIF file). This can be done more easily using the GUI GBond_Str and importing a CIF file that is automatically transformed into a CFL file.

\section{Example 2:}

A CFL file, called mfepo5.cfl, exists in the current directory; the program is invoked as follows:

Current_directory> Bond_Str mfepo5 <cr> $\cdots$

The screen output is the following:

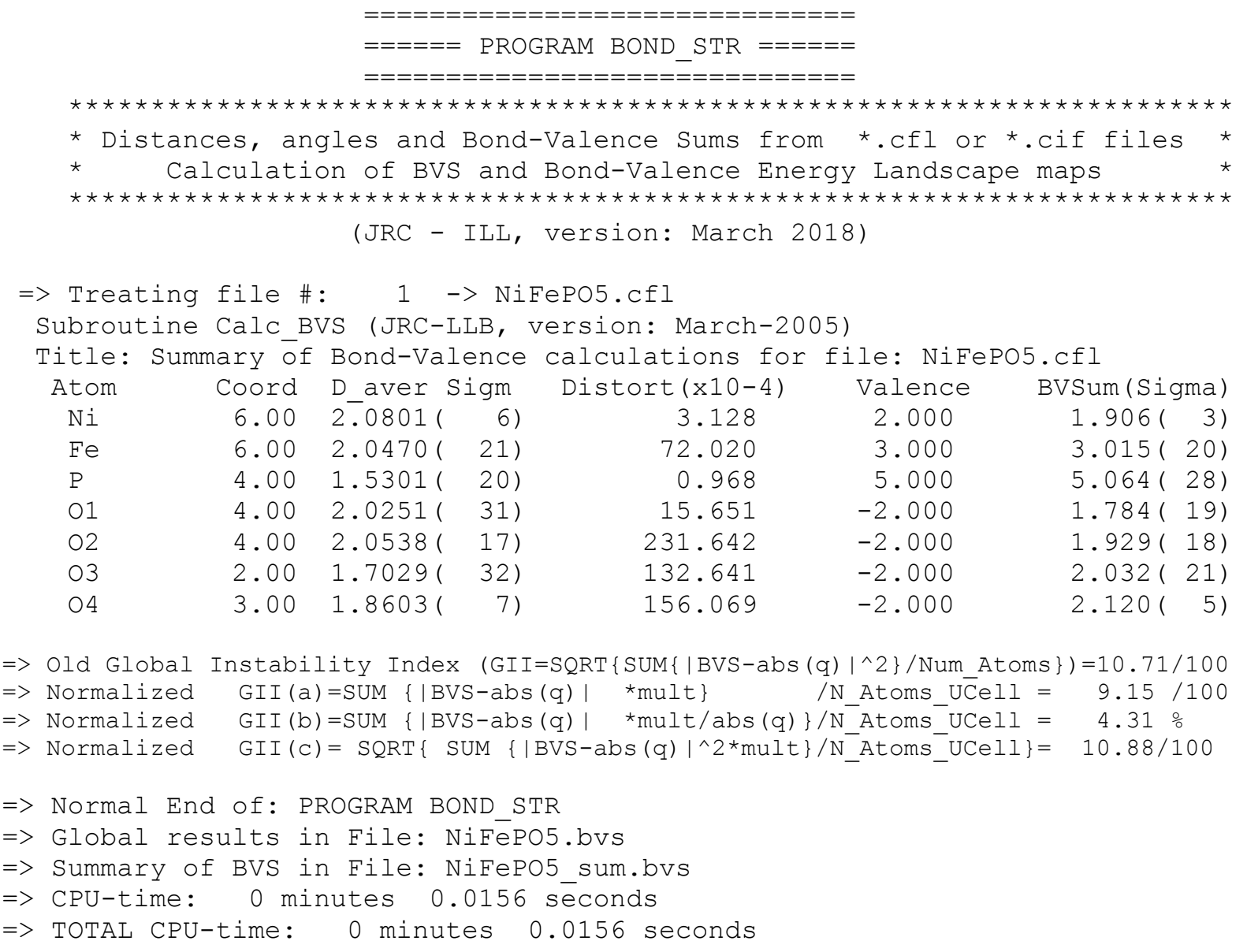

The information given in the screen output corresponds to the content of the summary file mfepo5_sum.bvs. All details are in the output file mfepo5.bvs. A CIF file is generated from the CFL file (NiFePO5_gen. cif) containing the relevant structural information. 
The content of the CFL corresponding to the above calculation is the following:

Start of the mfepo5. cfl file

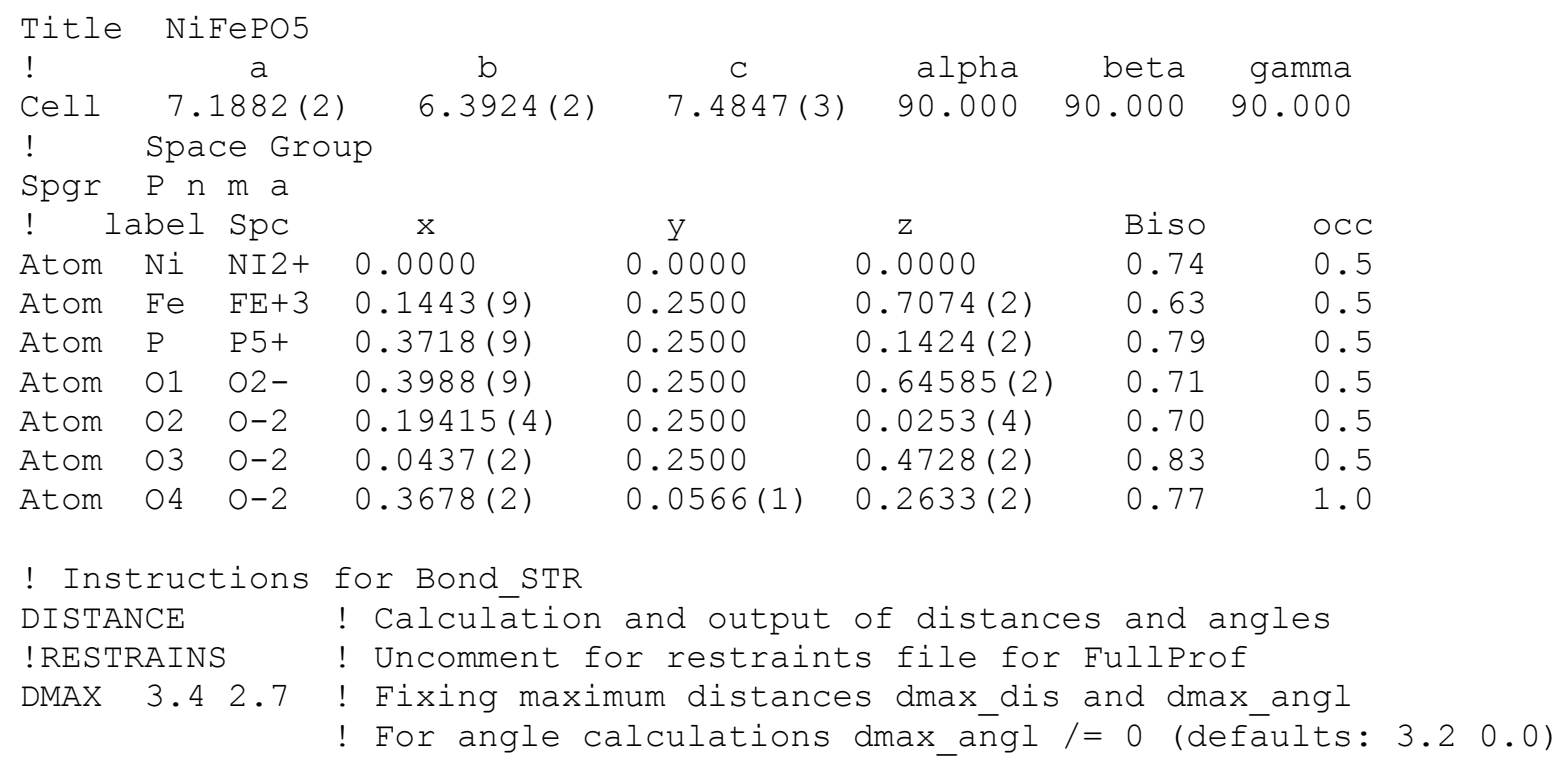

End of the mfepo5. cfl file

Notice the way of giving the oxidation state of the ions: the name of the element followed by $+/-n$ or $n+/-$ being $" n$ " the assumed valence. Notice also that the standard deviations can be given in parenthesis (as usual) but immediately following the last number. No space is permitted between the value and its standard deviation. The minimal set of keywords in a CFL file for being used as input of the program Bond_Str are: cell, spgr and atom. They are case insensitive.

The symbol "!" is used as a comment. The items following an atom keyword are: Label of the atom, element or species, fractional coordinates $\mathrm{x}, \mathrm{y}, \mathrm{z}$, isotropic displacement parameter (Biso) and occupation factor (proportional to the multiplicity of the site, e.g. $\circ \mathrm{CC}^{\mathrm{m}}=\mathrm{m} / \mathrm{M}$ ).

Two more items can be given: magnetic moment value and ionic charge as real values. If instead of the ionic species only the element symbol is provided the two additional items are needed: even if the magnetic moment is not used it should be given. An alternative atom-line corresponding to the first line in the above example can be written as follows:

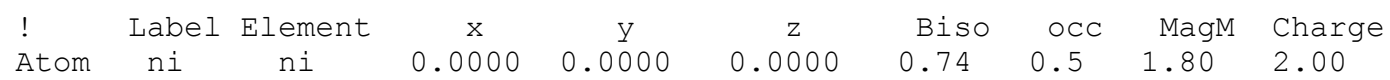

The keywords DISTANCE and RESTRAINS in the above examples do not need numerical values. They just instruct the program to change the output with respect to the default values.

The keyword DMAX is for limiting distance and angle calculations and it is followed by two real values (dmax_dis and dmax_angl).

Be careful not giving a high number for dmax_angle because the number of possible angles between three atoms that are at or below a distance dmax_angl increases strongly with dmax_angl. 
If user-given bond-valence parameters are to be provided, the instruction in the CFL file is as follows for a simple bond-valence sum calculation (for instance in the case of $\mathrm{La}^{3+}-\mathrm{O}^{2-}$ and $\left.\mathrm{Mn}^{3+}-\mathrm{O}^{2-}\right)$ :

$\begin{array}{llll}\text { BVPARM LA }+3 & 0-2 & 2.172 & 0.370\end{array}$

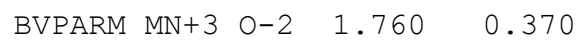

For calculating a bond valence energy landscape map, using softBVS parameters an average coordination must be given. For instance the line for $\mathrm{Mn} 3+$ should be written as:

$\begin{array}{lllll}\text { BVPARM MN+3 } & 0-2 & 1.760 & 0.370 & 6\end{array}$

In such a case the program calculates the Morse potential used in BVEL calculations from the BVS parameters provided in the BVPARM instruction. This is only true if the anions are in the internal list of chemical properties (ionic radius, electronegativity, etc.). Otherwise the user should provide the full list of parameters in the Morse potential using the instruction:

$\begin{array}{lrllcccc}\text { ! Cation } & \text { Anion } & \text { CN } & \text { Ro } & \text { Cutoff } & \text { D } & \text { Rmin } & \text { Alpha } \\ \text { BVELPAR MN+2 } & \text { N-3 } & 6 & 1.5 & 5 & 0.63394 & 1.81719 & 2.50000\end{array}$

For the meaning of the different parameters see the output file of BondStr for a case in which everything works with internal tables. The average coordination $\mathrm{CN}$ and the parameter Cutoff are not actually used for the calculations but should be provided for maintaining the format of the information provided in the output file.

In some cases the differences in the energies calculated by using softBVS parameters or using the most reliable internal tables (when the keyword SOFTBVS is not provided) may be important. The difference is less when one considers the activation energies, but it may attains values close to $1 \mathrm{eV}$. 


\section{Notes about the GUI GBond Str}

The program GBond_Str is a GUI for running Bond_Str without direct editing the input file. The interface has only a single window, except when the internal editor is invoked to

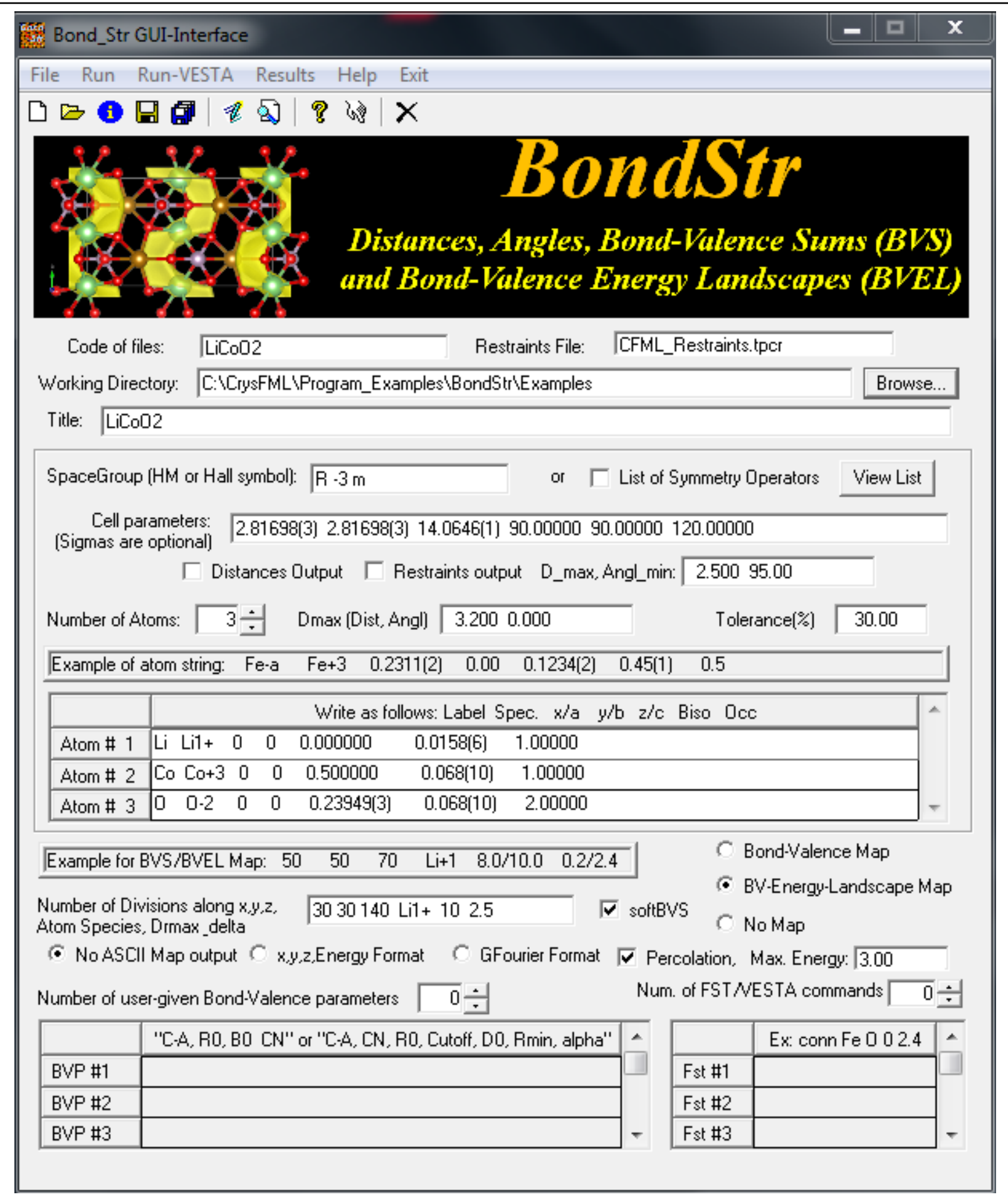

Figure 1: Screen shot of the GUI for Bond_Str after importing a CFL file. Notice that the calculation that will be performed correspond to a Bond-Valence Energy Landscape map followed by the estimation of the percolation energy.

visualise the results. 
The aspect of the interface after importing a CFL file is shown in Figure 1. The content of the CFL file is:

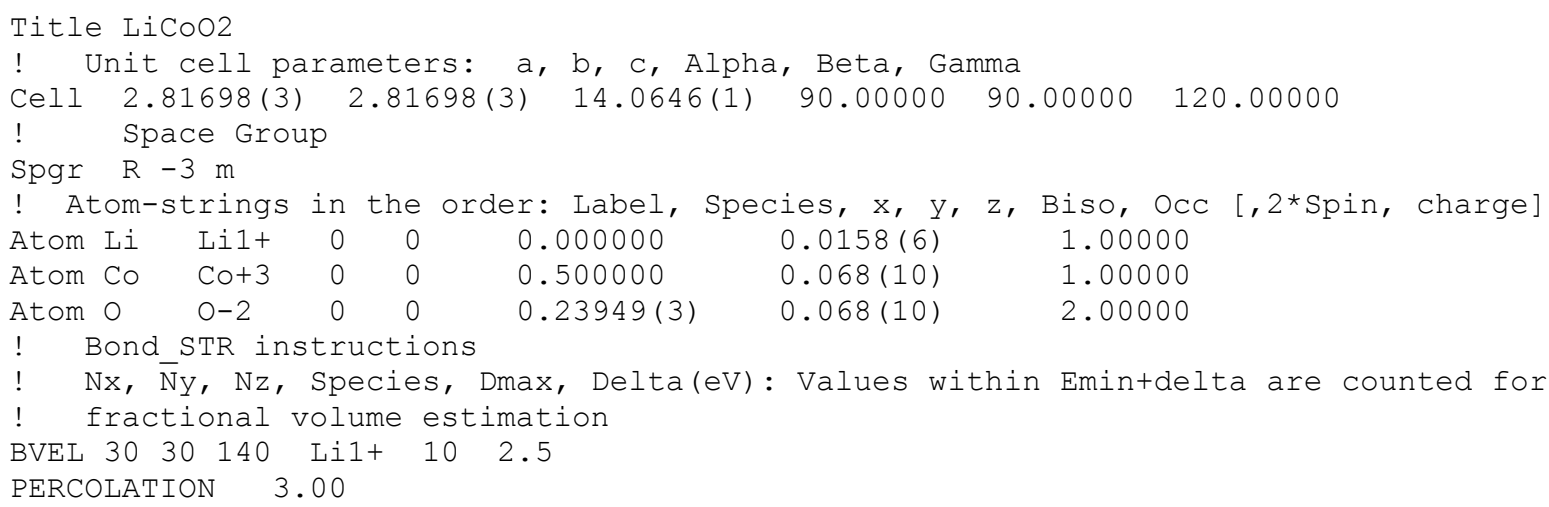

This interface to BondStr may be used to transform CIF files to CFL files with just a click as shown in Figure 2. In fact the previous CFL file has been converted from a CIF file. If the CIF files does not contain information about charges, the user may change that in the interface by completing the appropriate atom strings using a simple editor or by hand in GBond_Str.

Notice that the user can provide bond-valence parameters by filling the appropriate box that is

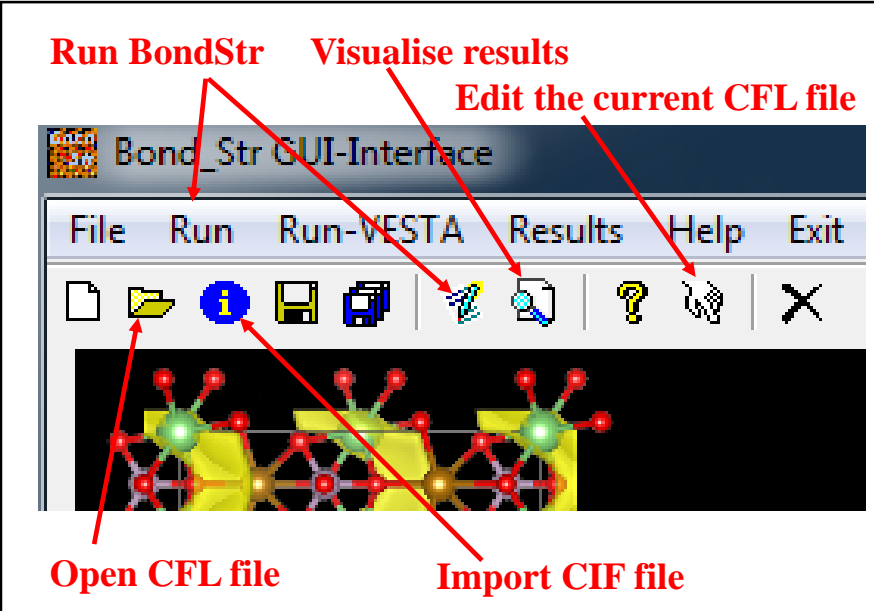

Figure 2: Some of the most important buttons of GBond_Str. activated as soon as one increases the number of user-given bond-valence parameter that is initially put to zero.

The program GBond_Str is just a tool for manipulating CIF and CFL files. There is no calculation inside GBond Str. When the user clicks on the run button (or select Run in the menu) the program saves the current CFL file and invokes Bond_Str with the code of the current file as argument. The normal output of Bond_Str is directed to the screen in a terminal console. As soon as Bond_Str finish the calculations GBond_Str takes the control and

edit the output file from Bond_str.

The program GBond_Str can be used to create the appropriate keywords for output of distance and angles, restraint files and the conditions for generating it. The keywords recognized by Bond_Str are TITLE, DISTAN, FST_CMD, BVPAR, DMAX, RESTDA, RESTR_FILE, SOFTBVS, TOL, RESTR, BVELPAR, PERCOLATION, MAP, BVEL, and VDIST.

The major part of these keywords (and the corresponding values) can be generated by GBond_Str, by clicking or checking the appropriate check boxes or radio buttons (the meaning of the keywords can be deduced from the text written in the GUI). However, VDIST can presently only be introduced in the CFL by hand, is VDIST is present the calculation of pair distributions of bond distances and volumes of polyhedra are calculated and output in the general output file of extension *. bvs. 
An example of the GUI content when the user provides directly the parameters defining the Morse potential for making a calculation of the BVEL map is given below in Fig. 3.

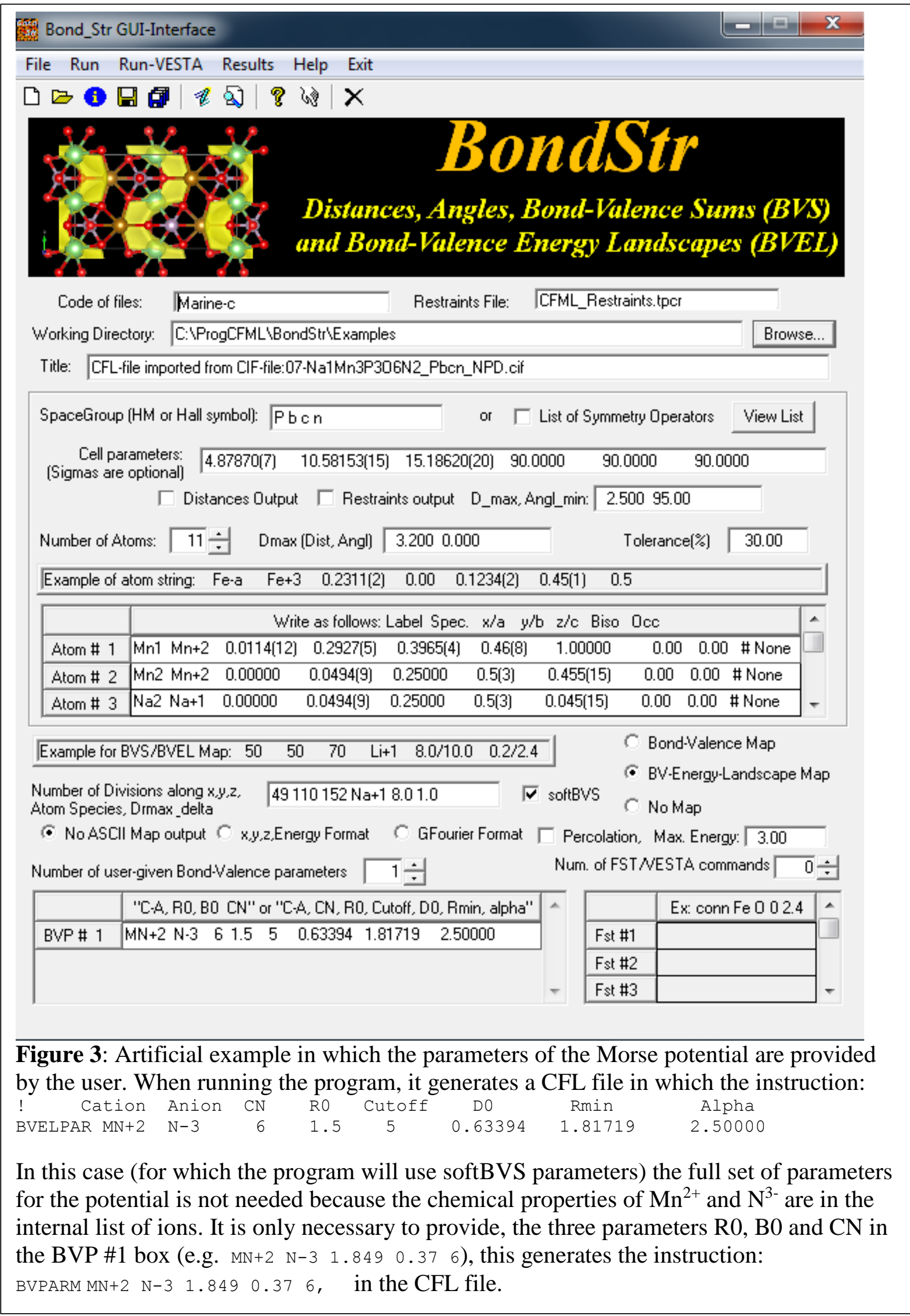




\section{The use of Bond_Str to treat many files in one single run}

The program Bond_Str can be used from a terminal to perform more complex data treatment. One can prepare a buffer file containing CIF or CFL files. The most interesting use of Bond_Str is when we have available a buffer file containing CFL files with oxidation states included in the structural information. This buffer file can be prepared using the program Formal_Charges. How to prepare this kind of file is described in the short manual of the program Formal_Charges. The program Bond_Str can be invoked in the command line giving the name of the buffer file as the first argument as:

$C: \backslash$ Database \CIFs>Bond_Str CfI_buffer.buf

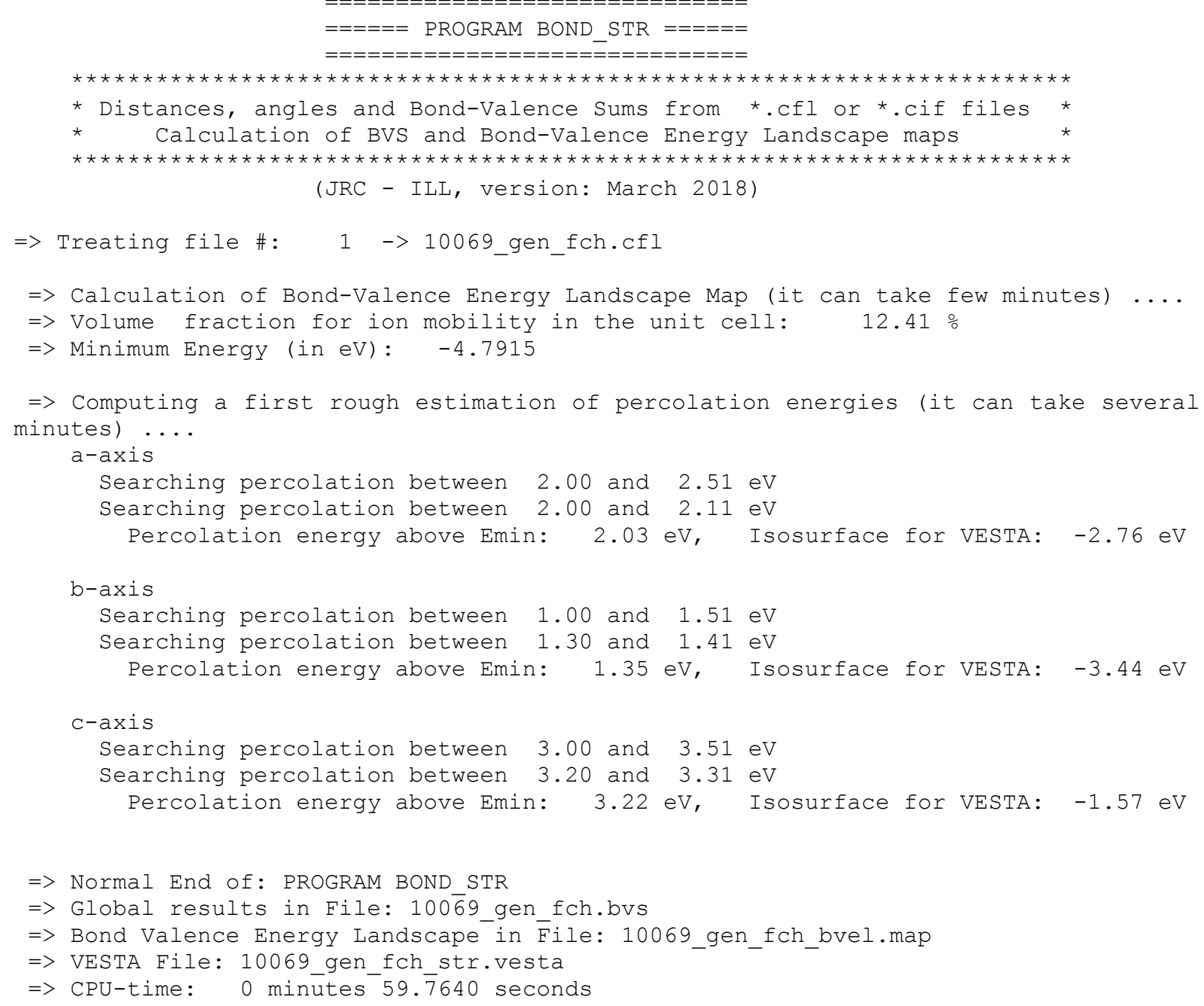




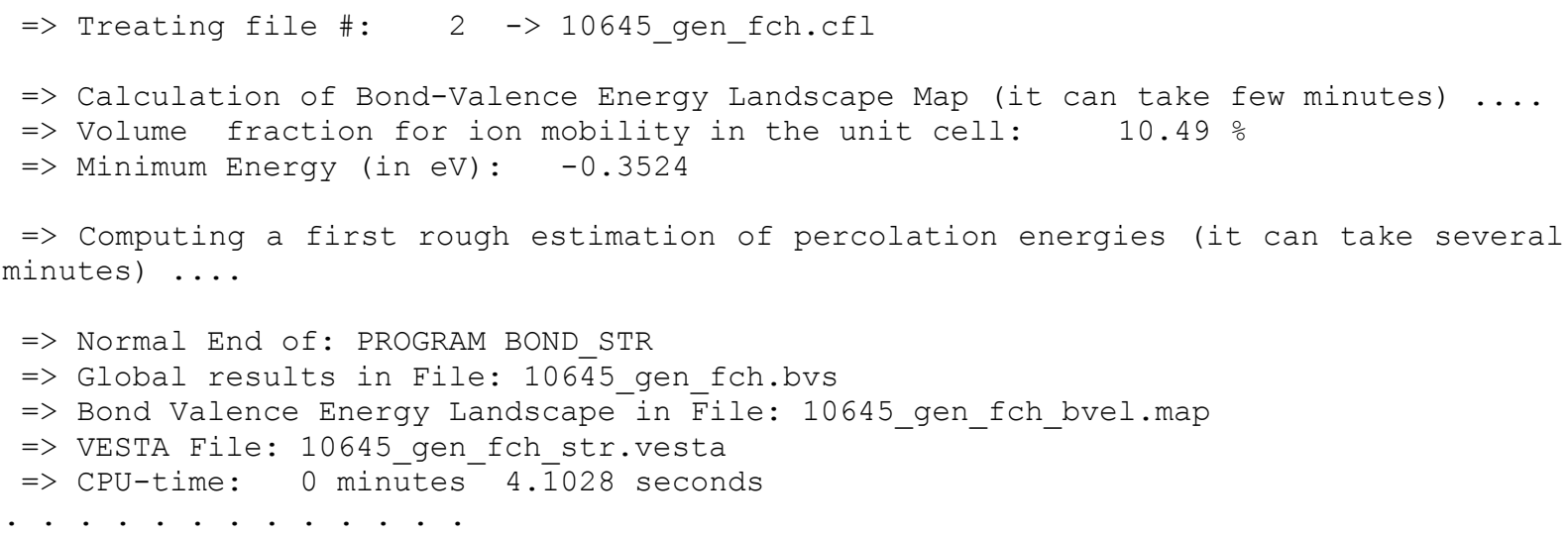

A part from the normal files generated by Bond_Str a global file called in this case cfl buffer glb.res containing a summary of the results. A rough output of this file in the screen can be obtained as:

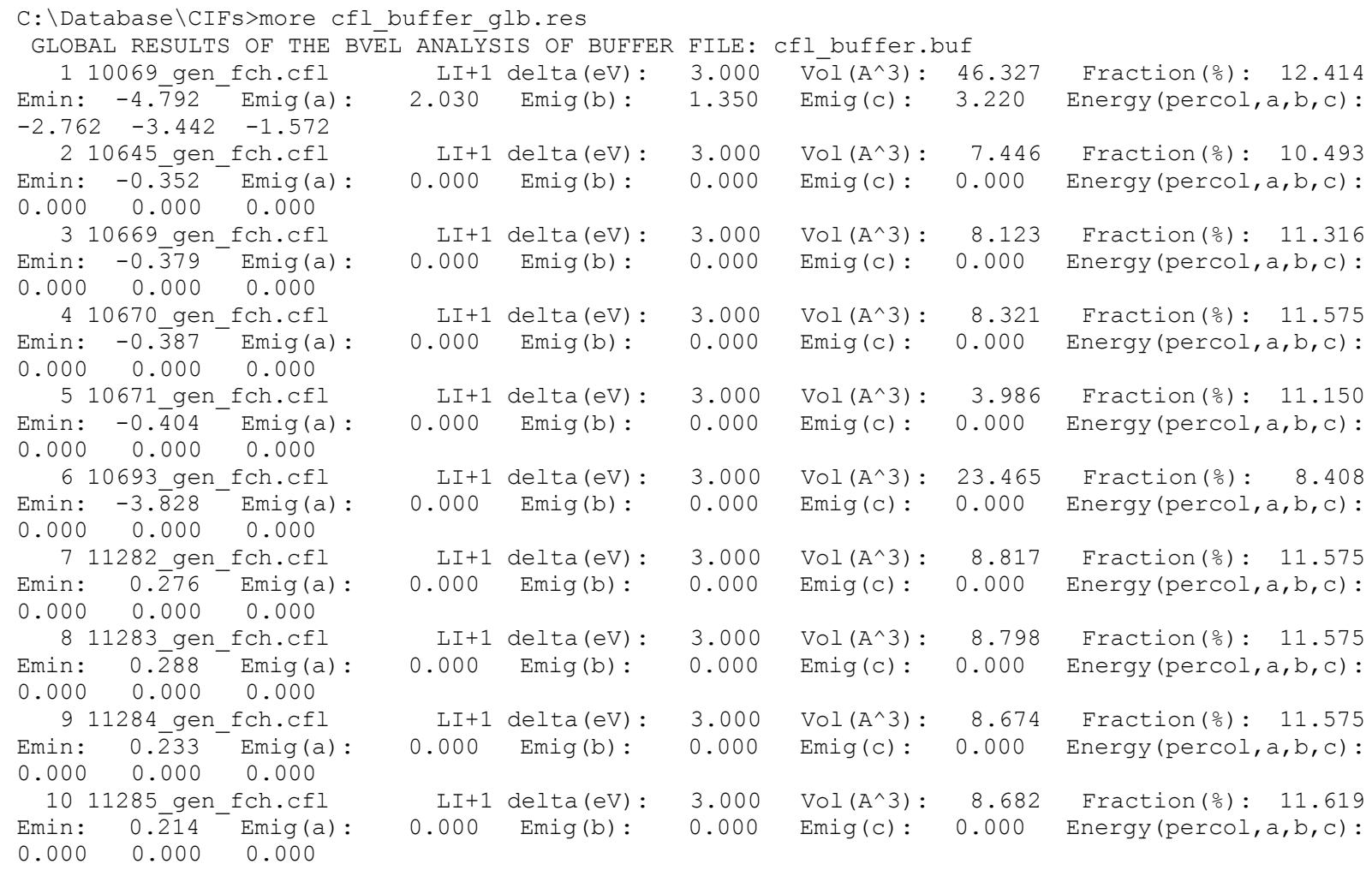

One can edit this file in order to see more clearly the content of the information that is included in a line per treated file. For each line there is the number in the sequence of the treated file, the name of the file, the mobile chemical species, the parameter delta in electron volts, the available volume for migration, the fraction of the total volume, the minimum energy, the migration (activation) energies (along $a, b$, and $c$ ) and the percolation energies (along $a, b$, and $c$ ). 
One can use a buffer file without calculation of the percolation/migration energies. For instance, if we are only interested in the calculation of bond-valence sums (using the same buffer file) one can invoke the program as:

C: \Database \CIFs>Bond_Str cfl_buffer.buf GII_only

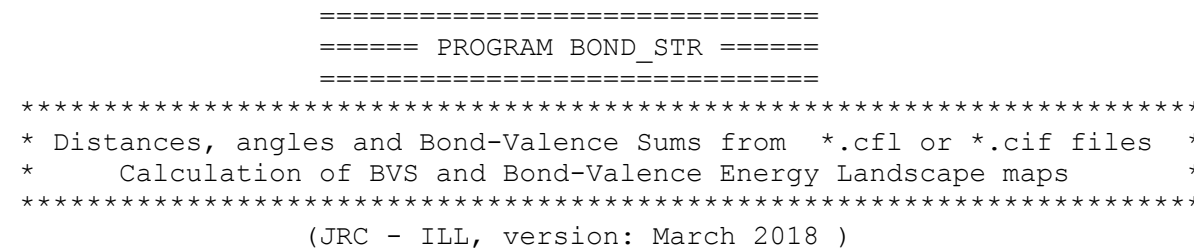

$=>$ Treating file\#: 1 -> 10069_gen_fch.cfl

Subroutine CalC BVS (JRC-LLB, version: March-2005)

Title: Summary of Bond-Valence calculations for file: 10069 gen fch.cfl

Atom Coord D_aver Sigm Distort(x10-4) Valence BVSum(Sigma)

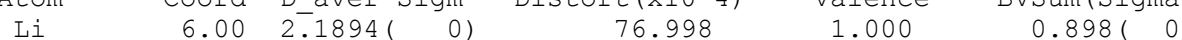

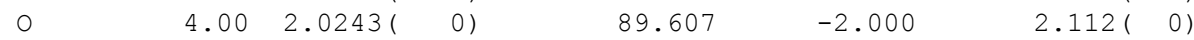

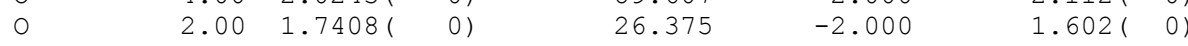

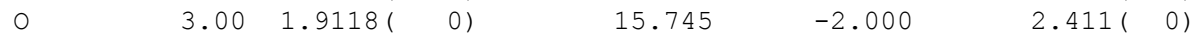

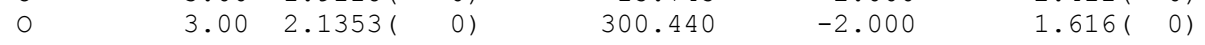

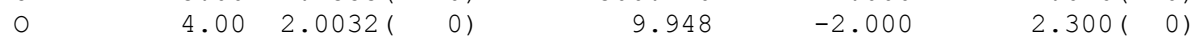

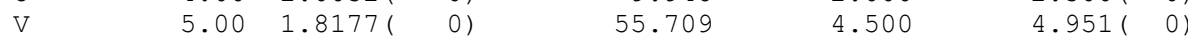

$\begin{array}{lllllll}\mathrm{V} & 5.00 & 1.9016( & 0 & 4.5302 & 4.497 & 4.500\end{array}$

$\Rightarrow$ Old Global Instability Index ( GII=SQRT $\{\operatorname{SUM}\{|\operatorname{BVS}-\mathrm{abs}(\mathrm{q})| \wedge 2\} / \operatorname{Num}$ Atoms $\})=33.28 / 100$

$\Rightarrow$ Normalized GII $(a)=\quad \operatorname{SUM}\left\{|\operatorname{BVS}-\mathrm{abs}(\mathrm{q})| *{ }^{*} \operatorname{mult}\right\} \quad / \mathrm{N}$ Atoms_UCell $=30.82 / 100$

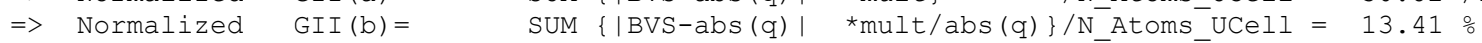

$\Rightarrow$ Normalized GII $(\mathrm{c})=\operatorname{SQRT}\left\{\operatorname{SUM}\{|\operatorname{BVS}-\mathrm{abs}(\mathrm{q})| \wedge 2 *\right.$ mult $\} \quad / \mathrm{N}^{-}$Atoms UCell $\}=33.28 / 100$

$=>$ Normal End of: PROGRAM BOND STR

$\Rightarrow$ Global results in File: $100 \overline{6} 9$ gen fch.bvs

$\Rightarrow$ Summary of BVS in File: 10069_gen fch_sum.bvs

$\Rightarrow$ CPU-time: 0 minutes 0.0156 seconds

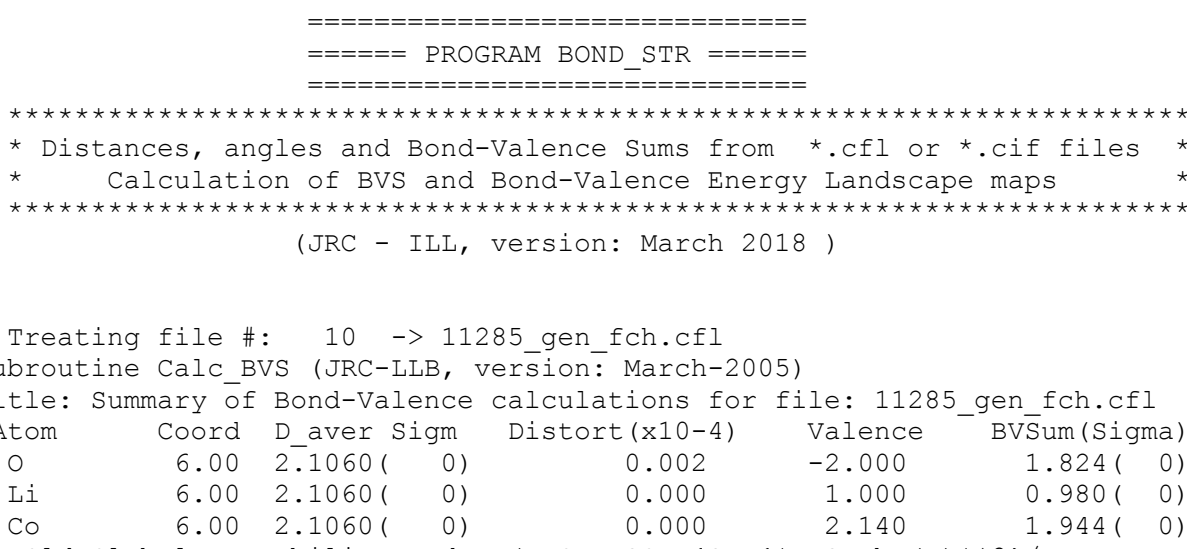

$\Rightarrow$ Old Global Instability Index ( GII=SQRT $\{\operatorname{SUM}\{\mid$ BVS-abs $(q) \mid \wedge 2\} /$ Num Atoms $\}=15.26 / 100$

$\Rightarrow$ Normalized GII $(a)=\quad \operatorname{SUM}\left\{|\mathrm{BVS}-\mathrm{abs}(\mathrm{q})|{ }^{*} \mathrm{mult}\right\} \quad / \mathrm{N}$ Atoms_UCel1 $=17.51 / 100$

$\Rightarrow$ Normalized GII $(\mathrm{b})=\quad \operatorname{SUM}\{|\mathrm{BVS}-\mathrm{abs}(\mathrm{q})| *$ mult/abs $(\mathrm{q})\} / \mathrm{N}_{-}^{-}$Atoms_UCel1 $=8.54 \%$

$\Rightarrow$ Normalized GII $(\mathrm{c})=\operatorname{SQRT}\left\{\operatorname{SUM}\left\{|\operatorname{BVS}-\mathrm{abs}(\mathrm{q})|^{\wedge}{ }^{*}{ }^{\star} \mathrm{mult}\right\} \quad\right.$ /N_Atoms_UCell $\}=17.99 / 100$

$=>$ Normal End of: PROGRAM BOND STR

$\Rightarrow$ Global results in File: $112 \overline{8} 5$ gen fch.bvs

$\Rightarrow$ Summary of BVS in File: 11285_gen fch_sum.bvs

$=>$ CPU-time: 0 minutes 0.0156 seconds

$=>$ TOTAL CPU-time: 0 minutes 0.3120 seconds 
Using the keyword GII only in the command line invocation of the program the cfl buffer_glb.res is simpler and contains only the value of the normalized GII ( $c$ ) and the name of the corresponding CFL file. For instance in the present case we have:

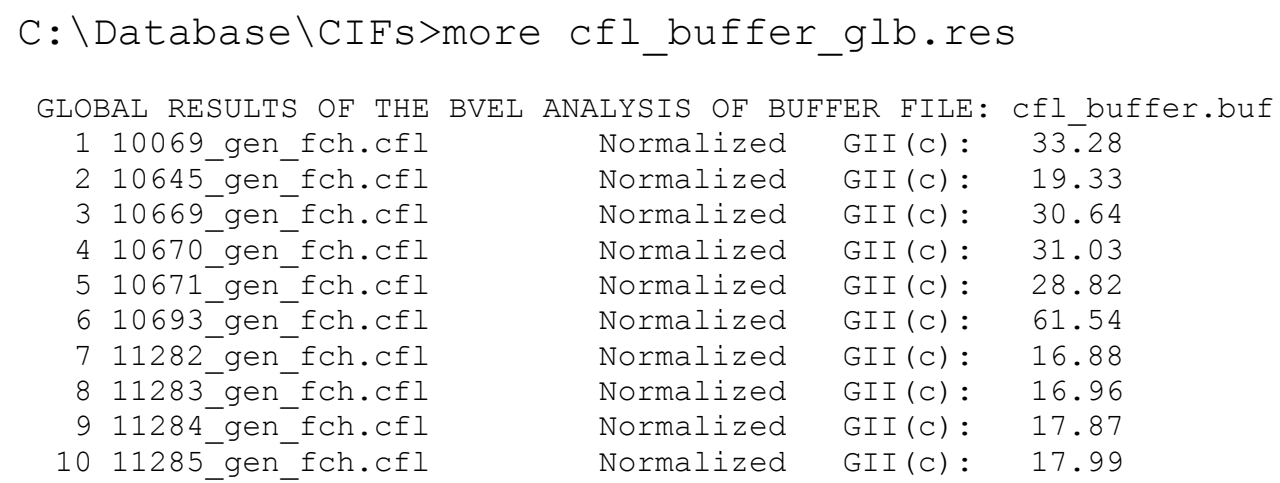

If we are only interested in the calculation of BVEL without calculating the percolation energies one can invoke the program as:

C: \Database \CIFS>Bond_Str Cfl_buffer.buf NPERCOLATION

The file cfl_buffer_glb.res is also simpler than in the general case, in our case it is as seen just below:

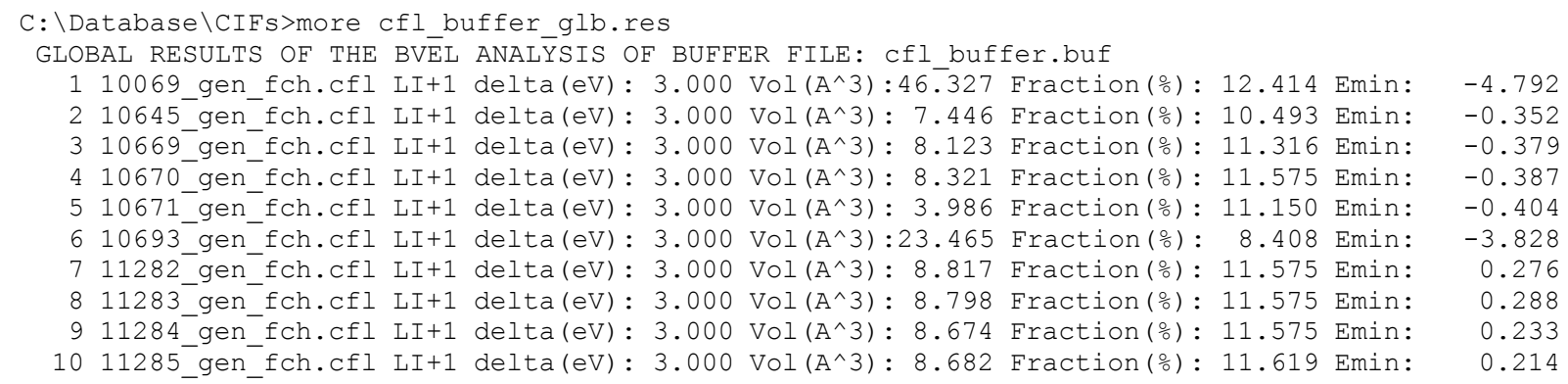

The information contained in the current document may be changed in forthcoming versions when more options will be available. More information about Bond_Str and its GUI can be obtained from the existing documentation in the FullProf Suite toolbar even if it is not currently updated. 